\title{
Luminosity function of low-mass X-ray binaries in the globular cluster system of NGC 1399
}

\author{
G. D’Ago ${ }^{1,2,3}$, M. Paolillo ${ }^{1,4,5}$, G. Fabbiano ${ }^{6}$, T. H. Puzia ${ }^{7}$, T. J.Maccarone ${ }^{8,9}$, A. Kundu ${ }^{10,11}$, \\ P. Goudfrooij ${ }^{12}$, and S. E. Zepf ${ }^{13}$
}

${ }^{1}$ Physics Dept., University of Napoli Federico II, via Cinthia 9, 80126 Napoli, Italy e-mail: paolillo@na.infn.it

2 Physics Dept. "E.R. Caianiello", University of Salerno, via Ponte Don Melillo, 84084 Fisciano, Italy

3 INFN, Gruppo collegato di Salerno, Sezione di Napoli, 84084 Fisciano, Italy

${ }^{4}$ INFN - Sezione di Napoli, via Cinthia 9, 80126 Napoli, Italy

5 ASI Science Data Center, Via del Politecnico s/n, 00133 Rome, Italy

${ }^{6}$ Harvard-Smithsonian Center for Astrophysics, 60 Garden St., Cambridge MA 02138, USA

7 Institute of Astrophysics, Pontificia Universidad Católica de Chile, Avenida Vicuña Mackenna 4860, 782-0436 Macul, Santiago, Chile

8 Department of Physics, Texas Tech University, Box 41051, Lubbock TX 79409-1051, USA

9 School of Physics and Astronomy, University of Southampton, Southampton SO17 1BJ, UK

10 TIFR, Homi Bhabha Road, 400005 Mumbai, India

11 Eureka Scientific Inc., 2452 Delmer St, Suite 100, Oakland CA 94602, USA

12 Space Telescope Science Institute, Baltimore MD 21218, USA

13 Department of Physics and Astronomy, Michigan State University, East Lansing MI 48824, USA

Received 20 September 2013 / Accepted 2 May 2014

\begin{abstract}
Aims. We present a study of the faint end of the X-ray luminosity function (XLF) of low-mass X-ray binaries (LMXBs) in the Globular Cluster (GC) system of the cD galaxy NGC 1399.

Methods. We performed a stacking experiment on 618 X-ray undetected GCs, in order to verify the presence of faint LMXBs and to constrain the faint-end slope of the GC-LMXBs XLF below the individual detection threshold of $8 \times 10^{37} \mathrm{erg} \mathrm{s}^{-1}$ in the $0.5-8 \mathrm{keV}$ band.

Results. We obtain a significant X-ray detection for the whole GC sample, as well as for the red and blue GC subpopulations, corresponding to an average luminosity per GC $\left\langle L_{\mathrm{X}}\right\rangle_{\mathrm{GC}}$ of $(3.6 \pm 1.0) \times 10^{36} \mathrm{erg} \mathrm{s}^{-1},(6.9 \pm 2.1) \times 10^{36} \mathrm{erg} \mathrm{s}^{-1}$, and $(1.7 \pm 0.9) \times$ $10^{36} \mathrm{erg} \mathrm{s}^{-1}$, respectively, for all GCs, red GCs, and blue GCs. If LMXBs in red and blue GCs have the same average intrinsic luminosity, we derive a red/blue ratio $\simeq 3$ of GCs hosting LMXBs $(2.5 \pm 1.0$ or $4.1 \pm 2.5$ depending on the surveyed region); alternatively, assuming the fractions observed for brighter sources, we measure an average X-ray luminosity of $L_{\mathrm{X}}=(4.3 \pm 1.3) \times 10^{37} \mathrm{erg} \mathrm{s}^{-1}$ and $L_{\mathrm{X}}=(3.4 \pm 1.7) \times 10^{37} \mathrm{erg} \mathrm{s}^{-1}$ per red and blue GC-LMXBs, respectively. In the assumption that the XLF follows a power-law distribution, we find that a low-luminosity break is required at $L_{\mathrm{X}} \leq 8 \times 10^{37} \mathrm{erg} \mathrm{s}^{-1}$ both in the whole, as well as in the color-selected (red and blue) subsamples. Given the bright-end slopes measured above the X-ray completeness limit, this result is significant at $>3 \sigma$ level. Our best estimates for the faint-end slope are $\beta_{L}=-1.39 /-1.38 /-1.36$ for all/red/blue GC-LMXBs. We also find evidence that the luminosity function becomes steeper at luminosities $L_{\mathrm{X}} \gtrsim 3 \times 10^{39} \mathrm{erg} \mathrm{s}^{-1}$, as observed in old ellipticals.

Conclusions. If most GCs host a single X-ray binary, we conclude that in NGC 1399 the XLF flattens at low luminosities as observed in other nearer galaxies, and we discuss some consequences of this flattening on LMXBs formation scenarios.
\end{abstract}

Key words. X-rays: binaries - X-rays: galaxies - galaxies: elliptical and lenticular, cD - galaxies: individual: NGC 1399

\section{Introduction}

$\mathrm{X}$-ray binaries (XRBs) are stellar binary systems consisting of a collapsed object (a neutron star or a black hole) accreting material from a donor star. In addition to being a probe of the physics of the accretion processes, $\mathrm{X}$-ray observations allow us to investigate the nature and evolution of stellar remnants and their host stellar systems. X-ray binaries contribute a significant fraction of the X-ray luminosity of galaxies, and the properties of the XRB population have been shown to be linked to the host galaxy type, its star formation history, galaxy environment, and merging history (see Fabbiano 1989, 2006). Several correlations have been found between the total X-ray luminosity from high-mass XRB (HMXB) and the galaxy star formation rate
(Ranalli et al. 3003; Gilfanov 2004; Hornschemeier et al. 2005; Lehmer et al. 2010; Mineo et al. 2012) as well as between the total emission from low-mass XRBs (LMXBs) and the galaxy stellar mass (Gilfanov 2004; Lehmer et al. 2010; Boroson et al. 2011; Zhang et al. 2012). Thus, XRBs can be used as a probe of the assembling process of galaxies over cosmic time (Fragos et al. 2013). Furthermore, the study of LMXBs probes the dynamics of dense stellar system, such as globular clusters since they most likely form via favorable multi-body encounters. For this reason, the understanding of their formation and evolutionary pathways also has strong implications for different types of studies such as the determination of the number of close binaries; these binaries can end up in a merging event and produce electromagnetic transients as well as gravitational waves. 
Given the old stellar population of elliptical galaxies their XRB population is represented by LMXBs. One of the primary tools we use to characterize the XRB population is the $\mathrm{X}$-ray luminosity function (XLF), which is dominated by XRBs in the luminosity range detectable in most external galaxies $\left(>10^{37} \mathrm{erg} \mathrm{s}^{-1}\right.$ ). The LMXBs luminosity function is steeper than that of young stellar systems (dominated by high-mass X-ray binaries and supernova remnants, see Fabbiano 2006, and references therein), and has two breaks: a high-luminosity break at $L_{\mathrm{X}} \geq 2 \times 10^{38} \mathrm{erg} \mathrm{s}^{-1}$ (Gilfanov 2004; Kim \& Fabbiano 2004; Humphrey \& Buote 2008), which may be absent in younger elliptical galaxies (Kim \& Fabbiano 2010), and a low-luminosity break at $L_{\mathrm{X}} \sim 5 \times 10^{37} \mathrm{erg} \mathrm{s}^{-1}$ (Voss \& Gilfanov 2006; Kim et al. 2009; Voss et al. 2009). The origin of these breaks is still debated, as they can be produced by differences in orbital period, mass ratio, type of donor/accreting star, or evolutionary stage of the XRBs population. Furthermore, in some elliptical galaxies such features are detected with low statistical significance (see Kim et al. 2006b) and incompleteness effects may be partly responsible for early reports of the low-luminosity break.

The discovery that in elliptical galaxies a large fraction of LMXBs (20\%-70\%) resides in globular clusters (GC; Angelini et al. 2001; Sarazin et al. 2003; Kim et al. 2006a; Paolillo et al. 2011) has triggered a debate about whether the GC and field populations of LMXBs share the same origin and properties. In these galaxies, these binary systems are more likely to be hosted in brighter, more compact and red (high metallicity) GCs, rather than fainter, less compact and blue (metal-poor) GCs (Bellazzini et al. 1995; Angelini et al. 2001; Kundu et al. 2002; Sarazin et al. 2003; Jordán et al. 2004; Kim et al. 2006a; Kundu et al. 2007; Sivakoff et al. 2008; Paolillo et al. 2011; Kim et al. 2013). The subpopulation of LMXBs in the field (field-LMXBs) and in globular clusters (GC-LMXBs) differs in spatial distribution, as the field-LMXBs follow the parent galaxy stellar distribution, while the GC-LMXBs follow the more extended GC distribution (e.g., Kundu et al. 2007; Paolillo et al. 2011); they also differ in the dependence on GC specific frequency $S_{N}$, since the number of LMXBs in GCs depends more strongly on $S_{N}$ than those in the field, as expected if not all binaries are originally formed within GCs (Kim et al. 2009; Paolillo et al. 2011; Mineo et al. 2014). Furthermore, at low luminosities the X-ray luminosity function of GC-LMXBs shows a more pronounced flattening than that of field-LMXBs because a lack of faint sources in GCs compared to the field (Kim et al. 2009; Voss et al. 2009; Zhang et al. 2011).

Given that long exposures are needed to study the lowluminosity XLF, these studies have been possible only for a handful of very close galaxies. This work is aimed at investigating the faint end of the XLF of GC-LMXBs in NGC 1399: the cD galaxy at the center of the Fornax cluster, which lies at a distance $D=20.1 \pm 0.4 \mathrm{Mpc}$ (Dunn \& Jerjen 2006). The GC-LMXB connection in NGC 1399 was studied in detail by Paolillo et al. (2011, hereafter P11) using combined Hubble Space Telescope (HST) and Chandra observations. This galaxy represents an ideal target for studying the GC-LMXBs XLF, since it has the highest fraction of GC-LMXBs among all known nearby galaxies, it is near enough to resolve GC sizes with the Advanced Camera for Surveys (ACS), and distant enough to sample efficiently the GC distribution out to large galactocentric radii.

In P11 the XLF of NGC 1399 was studied in the luminosity range from $\sim 8 \times 10^{37} \mathrm{erg} \mathrm{s}^{-1}$ up to $\sim 10^{39} \mathrm{erg} \mathrm{s}^{-1}$, where the XLF of both field- and GC-LMXBs is accurately approximated by a simple power-law, with marginal differences between the two populations (steeper XLF and lower median luminosity for field sources compared to GC). Below the lower luminosity limit, incompleteness effects complicate the detection of individual LMXBs. Here we use the same data as in P11 to perform a stacking experiment in order to probe the presence of faint GC-LMXBs, and to constrain the XLF slope at luminosities below the $8 \times 10^{37} \mathrm{erg} \mathrm{s}^{-1}$ and therefore study the dependence of the faint-end XLF on the color of the host GC.

\section{The dataset}

Our analysis is based on a combination of HST and Chandra observations. An extended discussion of the data properties and sample selection can be found in P11 and Puzia et al. (2014); here we briefly summarize the main properties of the dataset, and refer the reader to the latter references for more details.

\subsection{Optical data}

The optical data are based on observations with the HST Advanced Camera for Surveys (ACS; GO-10129, PI: T. Puzia) in the F606W filter. The observations were arranged in a $3 \times 3$ mosaic centered on the coordinates RA $(J 2000)=03^{\mathrm{h}} 38^{\mathrm{m}} 28.62^{\mathrm{s}}$ and Dec $(\mathrm{J} 2000)=-35^{\circ} 28^{\prime} 18.9^{\prime \prime}$ (see Fig. 1 in P11), so that the full mosaic extends out to a galactocentric radius of $\sim 50 \mathrm{kpc}$ from the galaxy center, $\sim 5.2$ effective radii of the diffuse galaxy light (de Vaucouleurs et al. 1991), and $\sim 4.9$ core radii of the globular cluster system density profile (Schuberth et al. 2010). Each field was observed for a total integration time of $2108 \mathrm{sec}-$ onds, and the individual exposures were combined into single images using the MultiDrizzle routine (Koekemoer et al. 2002), with a final pixel scale of $0.03^{\prime \prime} /$ pixel which, at the distance of NCG 1399, corresponds to $2.93 \mathrm{pc}\left(1^{\prime \prime}=97.7 \mathrm{pc}\right)$. The optical source catalog was created with the Source Extractor software (SExtractor, Bertin \& Arnouts 1996), and the astrometric solution was registered to the USNO-B1 catalog ${ }^{1}$, obtaining a final accuracy of $0.2^{\prime \prime}$ rms; globular clusters were selected as sources having a SExtractor stellarity index $\geq 0.9$ and $m_{V}(F 606 W)<26$ mag (in the VEGAmag system) in order to exclude extended sources and compact background galaxies. This results in a completeness of $>80 \%$ with $<10 \%$ contamination; we refer the reader to $\mathrm{P} 11$ for a detailed description of how these figures were derived, and to Brescia et al. (2012) for a discussion of alternative selection techniques.

We include photometry for our GCs, matching our catalog both with the Bassino et al. (2006) $C-T 1$ ground based GC catalog and the HST/ACS $g-z$ color catalog from Kundu et al. (2005). We adopted the following color and magnitude cuts to separate the different GC populations:

- Ground-based data: $1.0 \leq C-T 1<1.65$ for blue/metal-poor GCs and $1.65 \leq C-T 1<2.2$ for red/metal-rich GCs, and $T 1<23$ mag;

- HST data: $1.3 \leq g-z<1.9$ for blue/metal-poor GCs, $1.9 \leq$ $g-z<2.5$ for red/metal-rich GCs, with $z<22.5$ mag (in the VEGAmag system).

The $T 1$ and $z$ magnitude cuts were adopted to ensure a uniform completeness limit across the whole FOV, as discussed in more detail in P11.

\footnotetext{
http://tdc-www . harvard. edu/software/catalogs/ub1.
} html 


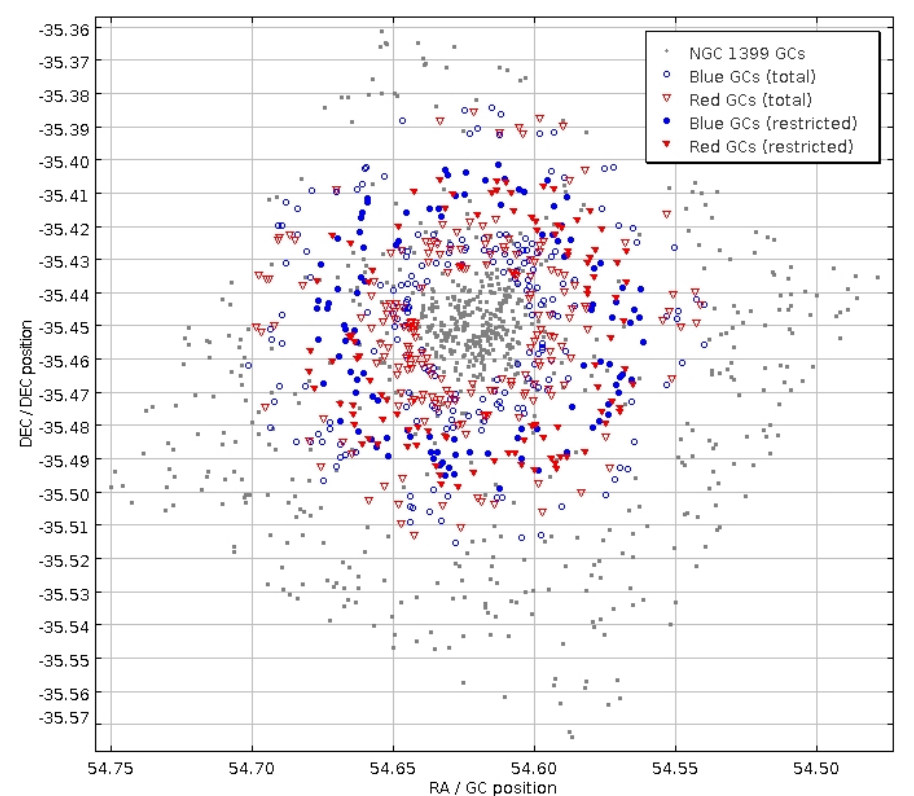

Fig. 1. Spatial distribution of the GCs used for the stacking experiments: blue circles and red triangles represent the total blue and red samples with no detected X-ray counterpart in P11. Solid symbols identify the restricted sample. Gray dots represent the rest of the GC system within the HST/ACS mosaic.

Table 1. Journal of Chandra observations.

\begin{tabular}{lccccc}
\hline \hline Obs. & Detector & Date & RA(J2000) & Dec(J2000) & $T_{\text {exp }}$ \\
\hline$\# 319$ & ACIS-S & 2000-Jan.-18 & $03^{\mathrm{h}} 38^{\mathrm{m}} 29.4^{\mathrm{s}}$ & $-35^{\circ} 27^{\prime} 00.4^{\prime \prime}$ & $56 \mathrm{ks}$ \\
$\# 4172$ & ACIS-I & 2003-May-26 & $03^{\mathrm{h}} 38^{\mathrm{m}} 25.6^{\mathrm{s}}$ & $-35^{\circ} 25^{\prime} 42.6^{\prime \prime}$ & $45 \mathrm{ks}$ \\
\hline
\end{tabular}

\subsection{X-ray data}

The X-ray data were retrieved from the Chandra public archive ${ }^{2}$. We used the observations with obsIDs No. 319 and No. 4172 (Table 1) for a total exposure time of $\sim 100 \mathrm{ks}$. The data were reduced with the CIAO software, extracting standard-grade events in the $0.3-8 \mathrm{keV}$ energy band and generating the corresponding exposure maps. As discussed in detail in P11, we used the WAVDETECT algorithm (Freeman et al. 2002) to assemble the X-ray source catalog, and the ACIS Extract software (AE, Broos et al. 2010) to derive the photometric parameters. The AE software is designed to deal with a combination of different observations, accounting for variations of source position inside the FOV and over the ACIS detector, using library templates of the ACIS PSF, as well as to improve the positional accuracy of our catalog. The final accuracy of the X-ray catalog is $0.33^{\prime \prime}$ with a maximum systematic offset of $0.6^{\prime \prime}$. The properties of all $230 \mathrm{X}$-ray sources detected individually in our observations are presented in detail in P11. In particular matching the optical and X-ray catalogs yielded $164 \mathrm{X}$-ray sources with optical counterparts within $1^{\prime \prime}$, out of which 136 are matched with GC candidates.

\section{GC sample selection}

As described in Sect. 1, the purpose of this work is to find clues about the properties of the GC-LMXB population below the threshold of $L_{\mathrm{X}} \sim 8 \times 10^{37} \mathrm{erg} \mathrm{s}^{-1}$. We tackle this problem by exploiting our optical catalog to stack the X-ray data at the

2 http://cxc.harvard.edu positions of X-ray undetected GCs. In order to maximize the efficiency of our stacking process, we have to take into account the main effects that contribute to lowering the $\mathrm{S} / \mathrm{N}$ ratio of faint LMXBs in our observations, i.e., the X-ray emission of the diffuse gaseous halo of NGC 1399 which mainly affects sources near the galaxy center, and the increase in PSF size with distance from the ACIS aimpoint which is dominant at large galactocentric radii. We thus limit our analysis to an annulus around the galaxy center with internal radius $r_{\mathrm{i}}=60^{\prime \prime}$ and external radius $r_{\mathrm{e}}=240^{\prime \prime}$ and consider only GCs with colors in the ranges discussed in Sect. 2.1, which additionally minimizes the contamination from background AGNs. To better constrain the XLF below the individual-source detection threshold (Sect. 5), we also defined a second smaller sample limited to GCs in the range $120^{\prime \prime}<r<180^{\prime \prime}$, which corresponds to the region with higher detection efficiency (and lower incompleteness at low luminosities) for point-like sources because of the minimization of the combined effects discussed above, as shown in Fig. 7 of P11.

In assembling the input GC position list for X-ray stacking, we excluded from our GC samples, all GCs individually detected in X-rays (Table 4 in P11). We also excluded from the list all GCs closer than $3^{\prime \prime}$ to an individually detected LMXB in order to avoid cross-contamination effects ${ }^{3}$. The two catalogs so selected consist of 618 GCs (of which 316 blue and 302 red) in the total annulus $\left(60^{\prime \prime}<r<240^{\prime \prime}\right)$ and of 210 GCs (of which 109 blue and $101 \mathrm{red})$ in the restricted annulus $\left(120^{\prime \prime}<r<180^{\prime \prime}\right)$. The $\mathrm{X}$-ray images centered on the input GCs were finally visually inspected to ensure that we did not include any source lurking just below the detection threshold. In Fig. 1 we show the spatial distribution of the GC samples used for the stacking experiment.

\section{X-ray stacking}

As done for individually detected sources, for our stacking experiment we used the AE software to derive the stacked photometry in the $0.5-8 \mathrm{keV}$ energy band for the sample of X-ray undetected GCs described in the previous section.

Given a list of source positions, AE models a polygonal extraction region which approximates the contour of the ChandraACIS PSF, taking into account the exact position within the detector; this region is used to measure the aperture photometry of the source and derive the total flux, after applying a correction for the encircled energy within the extraction area. In addition AE defines, for all nearby objects, a circular mask (with radius 1.1 times a radius that encloses $99 \%$ of the total flux) that is used to exclude the corresponding pixels from the estimation of the source flux and of the local background, in order to prevent the contamination due to close neighbors. This process is repeated for each observation separately, in order to account for the different position and exposure of every source in each image; the final flux is defined as an exposure-weighted average of the single measurements in each observation. Thus AE uses the appropriate PSF model, deriving the corresponding exposure map for each Chandra pointing separately, and generating masks that optimize the source and background extraction regions accounting for nearby objects. This procedure allows AE to be easily adapted to stacking analysis if we treat all sources as individual observations of the same object. In this case AE combines the individual photometric measurements, weighting them appropriately by their corresponding exposure time.

\footnotetext{
3 Within the considered spatial region, the ACIS PSF size is such that more than $90 \%$ of the flux is confined within this radius.
} 

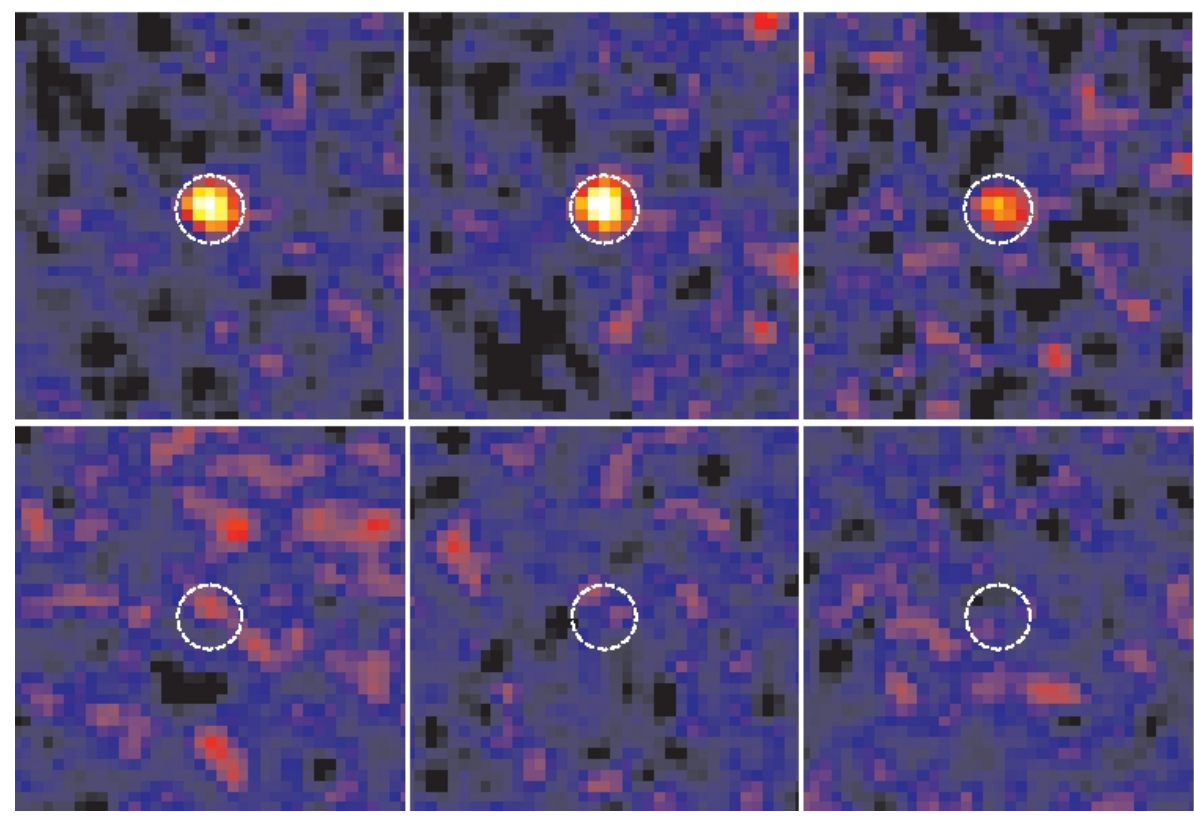

Fig. 2. Upper row: stacked images of all GCs subsamples: left) all 618 GCs; center) 316 red GCs; right) 302 blue GCs. The central circle highlights the $3^{\prime \prime}$ diameter region where most of the flux from the stacked LMXBs is concentrated. The images are produced through a sigmaclipped averaging algorithm with the clipping threshold set at $4 \sigma$. Lower row: same as the upper row, for 3 of the 100 samples simulated using random positions near real GCs (see discussion in text).

Table 2. Average flux and luminosity per GC, measured by the stacking experiment for all sources, as well as the red and blue subsamples, in the two different annular regions described in the text.

\begin{tabular}{lccc}
\hline \hline Sample & $\begin{array}{l}\text { No. of } \\
\text { sources }\end{array}$ & $\begin{array}{c}\left\langle f_{\mathrm{X}}\right\rangle_{\mathrm{GC}} \\
{\left[10^{-17} \mathrm{erg} \mathrm{cm}^{-2} \mathrm{~s}^{-1}\right]}\end{array}$ & $\begin{array}{c}\left\langle L_{\mathrm{X}}\right\rangle_{\mathrm{GC}} \\
{\left[10^{36} \mathrm{erg} \mathrm{s}^{-1}\right]}\end{array}$ \\
\hline \multicolumn{4}{c}{ Total sample: $60^{\prime \prime}<r<240^{\prime \prime}$} \\
All GCs & 618 & $13 \pm 1.6$ & $6.2 \pm 0.8$ \\
Red GCs & 302 & $19 \pm 2.4$ & $8.9 \pm 1.0$ \\
Blue GCs & 316 & $7.5 \pm 2.2$ & $3.6 \pm 1.0$ \\
\hline \multicolumn{4}{c}{ Restricted sample: $120^{\prime \prime}<r<180^{\prime \prime}$} \\
All GCs & 210 & $7.5 \pm 2.0$ & $3.6 \pm 1.0$ \\
Red GCs & 101 & $14 \pm 4$ & $6.9 \pm 2.1$ \\
Blue GCs & 109 & $3.6 \pm 1.9$ & $1.7 \pm 0.9$ \\
\hline
\end{tabular}

We repeated the stacking experiment six times: in each one of the two annuli discussed in Sect. 3, using the whole GC sample and, separately, for the blue and red populations. The X-ray fluxes and luminosities were derived assuming a bremsstrahlung emission $(k T=7 \mathrm{keV})$ with a photoelectric absorption component $N_{\mathrm{H}}=1.3 \times 10^{20} \mathrm{~cm}^{-2}$, which mimics the average spectrum of bright $\left(L_{X} \simeq 10^{38} \mathrm{erg} \mathrm{s}^{-1}\right)$ LMXBs (see P11). The average energy fluxes and luminosities are summarized in Table 2. The table shows that we are able to detect an X-ray signal with a significance of $>7.5 \sigma, 9 \sigma$, and $>3.5 \sigma$ for the whole, red, and blue subsamples, respectively, in the total sample; similarly for the restricted sample the significances are $>3.5 \sigma,>3 \sigma$, and $\sim 2 \sigma$. Furthermore, we find that the average X-ray luminosity of red GCs is larger than that of the blue GCs with more than $3.5 \sigma$ and $2 \sigma$ confidence, respectively, for the total and restricted samples.

In Fig. 2 (upper row) we show the stacked images of the neighborhood of each of the three color subsamples for the annulus $60^{\prime \prime}<r<240^{\prime \prime}$, obtained computing the average value per pixel after applying a sigma clipping threshold of $4 \sigma$. These images are presented here only to visualize the results of the stacking process, since the actual flux measurements shown in Table 2 are derived by AE through the more accurate procedure discussed above. As an additional verification of our method, we measured the total flux from the images in Fig. 2, finding that the results are fully consistent with those produced by AE. Each input GC position was also visually inspected to make sure that there is no evident $\mathrm{X}$-ray source lurking just below the detection threshold used by P11.

In order to test the robustness of our results, we performed 100 simulations repeating the stacking process at random positions around each GC. Each simulation contained as many simulated positions as the actual dataset; these positions were placed between $3^{\prime \prime}$ and $15^{\prime \prime}$ from the actual GCs in order to preserve the source and background spatial distributions, preventing any contamination from the GCs themselves. As done for the real stacking experiment, we excluded any position closer than $3^{\prime \prime}$ to a detected X-ray source (whose pixels are masked by the AE algorithm) and we forced the simulated positions to be at least $3^{\prime \prime}$ apart to make sure that each simulation represents an independent dataset. We find an average stacked luminosity of $L_{\mathrm{X}}=$ $(1.6 \pm 2.6) \times 10^{38} \mathrm{erg} \mathrm{s}^{-1}$, i.e. $\left\langle L_{\mathrm{X}}\right\rangle_{\mathrm{GC}}=(0.8 \pm 1.2) \times 10^{36} \mathrm{erg} \mathrm{s}^{-1}$ consistent with a null flux, thus confirming for real GCs a significant detection at $99 \%$ level. Some examples of the result of the stacking experiment for the simulated sample is shown in the lower panel of Fig. 2.

Since our stacking process only allows us to derive average luminosities, to compare our result with the properties of the brighter population of sources detected individually in P11, we need to make some assumptions about the distribution of LMXBs within GCs. Assuming that red and blue GC-LMXBs have the same average $X$-ray luminosity distribution function, the relative number of red $\left(\phi_{\text {red }}^{\mathrm{GC}-\mathrm{LMXB}}\right)$ and blue $\left(\phi_{\text {blue }}^{\mathrm{GC}-\mathrm{LMXB}}\right) \mathrm{GCs}$ hosting a LMXB, for our total sample, is given by

$\frac{\phi_{\text {red }}^{\mathrm{GC}-\mathrm{LMXB}}}{\phi_{\text {blue }}^{\mathrm{GC}-\mathrm{LMXB}}}=\frac{L_{\text {red }}^{\text {stack }}}{n_{\text {red }}} \frac{n_{\text {blue }}}{L_{\text {blue }}^{\text {stack }}}=\frac{\left\langle L_{\mathrm{X}}\right\rangle_{\text {redGC }}}{\left\langle L_{\mathrm{X}}\right\rangle_{\text {blueGC }}}=2.5 \pm 1.0$, 
where $L_{\text {red }}^{\text {stack }}$ and $L_{\text {blue }}^{\text {stack }}$ are, respectively, the stacked luminosities obtained from the red and blue GC subsamples, while $n_{\text {red }}$ and $n_{\text {blue }}$ are the number of the GCs in such subsamples. A similar calculation for the restricted sample yields

$$
\frac{\phi_{\text {red }}^{\mathrm{GC}-\mathrm{LMXB}}}{\phi_{\text {blue }}^{\mathrm{GC}-\mathrm{LMXB}}}=4.1 \pm 2.5 \text {. }
$$

In P11 we found that the fractions of red $\left(\phi_{\text {red }}^{\mathrm{GC}-\mathrm{LMXB}}=16 \%\right)$ and blue $\left(\phi_{\text {blue }}^{\mathrm{GC}-\mathrm{LMXB}}=5 \%\right)$ GCs hosting a LMXB lead to a ratio $\phi_{\text {red }}^{\mathrm{GC}-\mathrm{LMXB}} / \phi_{\text {blue }}^{\mathrm{GC}-\mathrm{LMXB}}=3.2$. The ratio for the stacked sample is thus consistent within $1 \sigma$ with the ratios observed at higher luminosities (and over the whole galaxy) in NGC 1399, and in agreement with the 3:1 ratio usually reported in the literature for other elliptical galaxies (Kundu et al. 2002; Jordán et al. 2004; Kim et al. 2006a; Kundu et al. 2007; Sivakoff et al. 2008; Kim et al. 2013).

Conversely, fixing the relative fractions $\phi_{\text {red }}^{\mathrm{GC}-\mathrm{LMXB}}$ and $\phi_{\text {blue }}^{\mathrm{GC}-\mathrm{LMXB}}$ to the values observed at brighter luminosities, we find that faint red and blue GC-LMXBs in the total sample, have compatible average luminosities:

$$
\begin{aligned}
& \bar{L}_{\text {red }}=\frac{L_{\text {red }}^{\text {stack }}}{n_{\text {red }} \cdot \phi_{\text {red }}^{\mathrm{GC}-\text { LMXB }}}=(5.5 \pm 0.7) \times 10^{37} \mathrm{erg} \mathrm{s}^{-1} \\
& \bar{L}_{\text {blue }}=\frac{L_{\text {blue }}^{\text {stack }}}{n_{\text {blue }} \cdot \phi_{\text {blue }}^{\mathrm{GC}-L M X B}}=(7.2 \pm 2.0) \times 10^{37} \mathrm{erg} \mathrm{s}^{-1} .
\end{aligned}
$$

The same holds for the restricted sample, where:

$\bar{L}_{\text {red }}=(4.3 \pm 1.3) \times 10^{37} \mathrm{erg} \mathrm{s}^{-1}$

$\bar{L}_{\text {blue }}=(3.4 \pm 1.7) \times 10^{37} \mathrm{erg} \mathrm{s}^{-1}$.

In both cases, the average luminosity is consistent with an emission below the completeness limit of the (uncorrected) X-ray data of $L_{X} \sim 2 \times 10^{38} \mathrm{erg} \mathrm{s}^{-1}$ found in P11. Moreover, for the restricted sample where, as discussed before, the source catalog reaches fainter completeness levels, the stacked luminosities are lower than the faintest source found in P11 with $L_{\mathrm{X}} \sim 5 \times 10^{37} \mathrm{erg} \mathrm{s}^{-1}$, as expected if we are detecting the cumulative emission due to LMXBs below our individual source detection threshold.

\section{The GC-LMXBs luminosity function}

As discussed in the introduction, previous studies have found that the GC-LMXBs X-ray luminosity function in elliptical galaxies can be represented by a double power law with a break at $\sim 5 \times 10^{37} \mathrm{erg} \mathrm{s}^{-1}$. This result is limited so far only to a few nearby galaxies, for which very long exposures enable the detection of individual LMXBs down to X-ray luminosities of $10^{35} \mathrm{erg} \mathrm{s}^{-1}$. Our stacking analysis allows us to verify whether this result also holds for a giant elliptical like NGC 1399. To this end we parametrize the XLF as

$\mathrm{d} N / \mathrm{d} L=\Phi(L)= \begin{cases}A \cdot\left(L / L_{\mathrm{bk}}\right)^{\beta_{L}} & \text { if } L<L_{\mathrm{bk}} \\ A \cdot\left(L / L_{\mathrm{bk}}\right)^{\beta_{\mathrm{H}}} & \text { if } L \geq L_{\mathrm{bk}},\end{cases}$

where $A$ is the normalization of the XLF at the break luminosity $L_{\mathrm{bk}}$, while $\beta_{L}$ and $\beta_{\mathrm{H}}$ are, respectively, the faint-end slope and the bright-end slope of the XLF.

The XLF of bright, individually detected sources was already presented in $\mathrm{P} 11$; here we refine the analysis, considering the two radial annuli used to define the total and restricted samples in Sect. 3. We point out that for the study of the features of the
XLF, the restricted sample allows more stringent constraints to be derived than the total one does. As noted before, the annulus $120^{\prime \prime}<r<180^{\prime \prime}$ represents the region less affected by incompleteness problems that allows us to probe the X-ray population down to fainter luminosities. For this reason we start by discussing the restricted sample, and later compare the results to those obtained from the wider annulus. To this end we first fitted the luminosity function of the individually detected sources in the $120^{\prime \prime}-180^{\prime \prime}$ range, with a power-law function; we used the maximum likelihood (ML) plus the Kolmogorov-Smirnov (KS) approach described by Clauset et al. (2009) and Newman (2005) in order to derive both the power-law slope, its uncertainty, and the minimum luminosity above which the data follow a power-law distribution. We obtain a differential XLF bestfit slope of $\beta_{\mathrm{H}}=-2.4 \pm 0.3$ above a minimum luminosity of $L_{\mathrm{X}}=8 \times 10^{37}$ erg s$^{-1}$ (Fig. 3).

We note that this slope is steeper than the one found for the total GC XLF in P11, which had $\beta_{\mathrm{H}}=-1.7 \pm 0.2$. However this discrepancy (always consistent within the errors) can be explained as a combination of different factors: first of all the total XLF suffers from problems with completeness at the faint end because of the problems discussed in Sect. 3 which tend to produce a flatter distribution; while a completeness correction was implemented in P11, residual effects are likely still present. Second, in the case of the total XLF the fitting method used was a simple $\chi^{2}$ because of the need to correct for AGN contamination (an effect which is not significant here because of the smaller area), and this generally results in lower accuracy fits. Some residual AGN contamination was also possibly present at the bright end since the total sample was not color-selected as it was here (see discussion in P11), again resulting in a flatter XLF slope. Finally, we cannot exclude that the XLF is intrinsically different at different galactocentric distances. We thus adopt here the $\beta_{\mathrm{H}}=-2.4$ value obtained from the ML fit.

We did not detect a break in P11, although the GC XLF seems to flatten below $\sim 8 \times 10^{37} \mathrm{erg} \mathrm{s}^{-1}$; as discussed above, completeness effects may be partly responsible for the flattening. A similar bend is observed here for the $120^{\prime \prime}-180^{\prime \prime}$ XLF. We thus conservatively assume that $L_{\mathrm{bk}}=8 \times 10^{37} \mathrm{erg} \mathrm{s}^{-1}$, and we will discuss below the consequences of relaxing this hypothesis. We note that this break value is also supported by the KS test performed to identify in what luminosity range a power law function provides the best fit.

The faint-end slope $\beta_{L}$ is determined requiring that the integral of the XLF below the break is equal to the total measured luminosity for $L<L_{\mathrm{bk}}$. The latter is the sum of two components: the stacked luminosity $L_{\text {stack }}=\left\langle L_{\mathrm{X}}\right\rangle_{\mathrm{GC}} \cdot n_{\mathrm{GC}}$ presented in Table 2, and the residual contribution $L_{\mathrm{faintLMXB}}$ of the seven individually detected LMXBs with $5 \times 10^{37} \mathrm{erg} \mathrm{s}^{-1}<L<L_{\mathrm{bk}}$

$$
\int_{10^{32}}^{8 \times 10^{37}} \Phi(L) L \mathrm{~d} L=L_{\text {stack }}+L_{\text {faintLMXB }},
$$

where for the lower integration limit we adopted the average luminosity of a quiescent binary star $L=10^{32} \mathrm{erg} \mathrm{s}^{-1}$ (Verbunt \& Lewin 2004); however, even if we integrate the function down to $L_{\mathrm{X}}=0$, our results do not change significantly. The results, for all the subsamples discussed in the previous section, are summarized in Table 3 while in Fig. 3 is shown the differential GC XLF $\Phi(L)$ and its $3 \sigma$ limits. The effect of the $12 \mathrm{GCs}$ that we removed due to the overlap with nearby LMXBs (Sect. 3) would produce a slight bias in the determination of the faint-end slope, but we verified that, assuming that they have the same properties and average X-ray luminosity of the rest of the stacked GC sample, the faint-end slope would increase by only $\sim 1 \%$. 

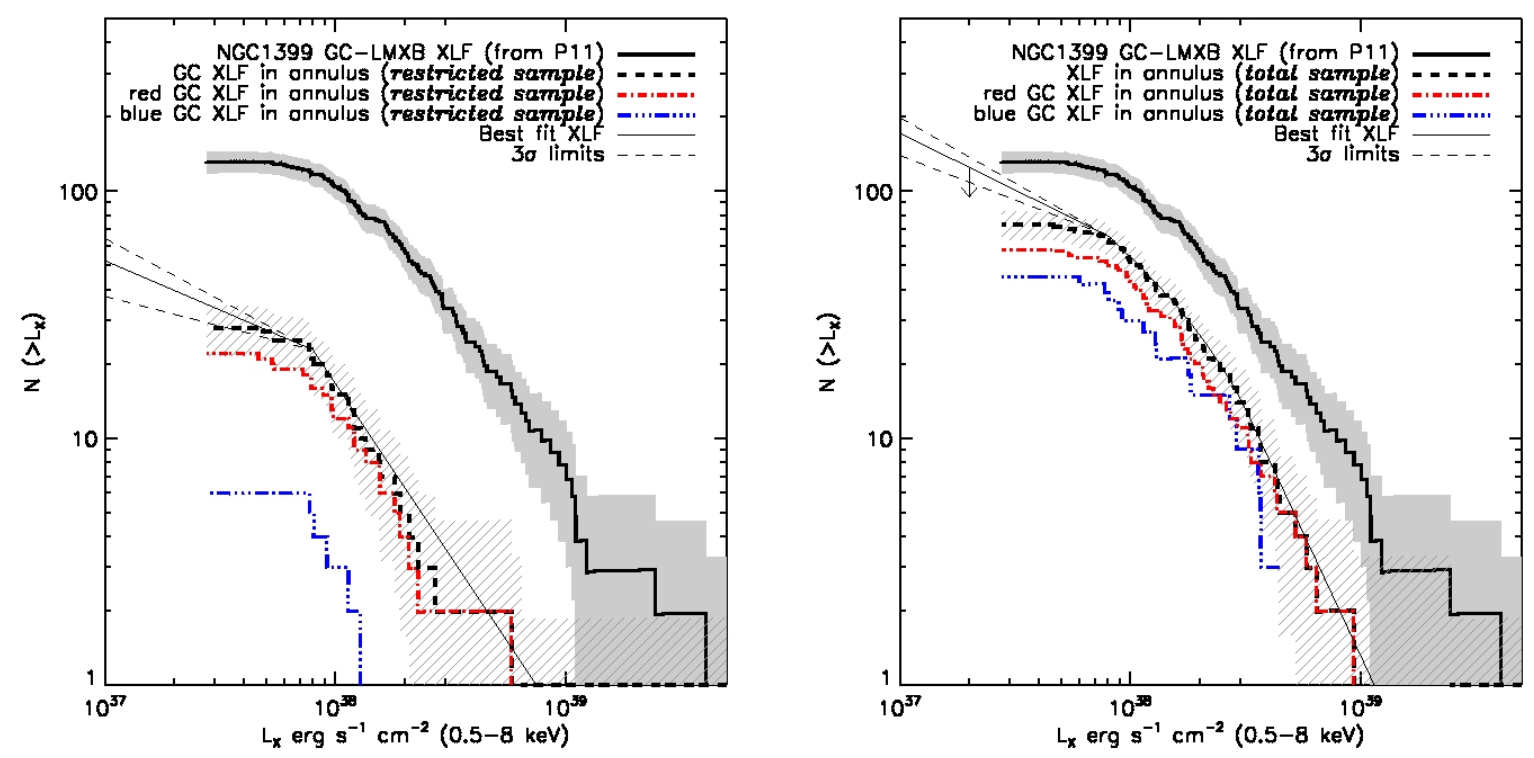

Fig. 3. Left panel: cumulative XLF for all, red, and blue (individually detected) GCs-LMXB within the $120^{\prime \prime}-180^{\prime \prime}$ annulus (restricted sample). For comparison the thick solid line represents the total XLF in NGC 1399, as derived in P11. The statistical uncertainties are shown, for the total NGC 1399 and annular XLF, as shaded (solid and dashed) areas. For clarity, the best-fit analytical XLF (according to Eq. (5)), is shown only for the total GCs-LMXB annular sample as a thin solid line, while its upper and lower $3 \sigma$ limits (Table 3 ) are marked by dashed lines. Right panel: as left panel for the total sample. The downward arrow emphasizes that in this case the faint-end slope has to be considered a conservative limit (see discussion in the text).

Table 3. Differential slope $\beta_{L}$ of the faint end of the GC XLF for the total and restricted samples, derived from Eq. (6), with upper and lower $3 \sigma$ limits.

\begin{tabular}{|c|c|c|c|}
\hline & $\beta_{L}$ & $\beta_{L}+3 \sigma$ & $\beta_{L}-3 \sigma$ \\
\hline \multicolumn{4}{|c|}{ Total sample: $60^{\prime \prime}<r<240^{\prime \prime}$} \\
\hline All GCs & -1.46 & -1.36 & -1.53 \\
\hline Red GCs & -1.44 & -1.36 & -1.50 \\
\hline Blue GCs & -1.53 & -1.23 & -1.67 \\
\hline \multicolumn{4}{|c|}{ Restricted sample: $120^{\prime \prime}<r<180^{\prime \prime}$} \\
\hline All GCs & -1.39 & -1.23 & -1.49 \\
\hline Red GCs & -1.38 & -1.21 & -1.48 \\
\hline Blue GCs & -1.36 & indef. ${ }^{a}$ & -1.54 \\
\hline
\end{tabular}

Notes. ${ }^{(a)}$ The lower limit on the differential XLF slope of blue GCs in the restricted sample is undefined since the stacking results are compatible within $3 \sigma$ with a null flux and a flat cumulative XLF.

The results show that the XLF flattens significantly for $L<$ $L_{\mathrm{bk}}$, and that even at the $3 \sigma$ confidence level the GC XLF slope is incompatible with the bright-end slope $\left(\beta_{\mathrm{H}}=-2.4\right)$. This conclusion still holds even considering separately the red and blue subsamples; this is not surprising for red GCs which represent the bulk of the population, while for blue GCs the result is confirmed even though that the uncertainties are larger as a result of the poor statistics. In this latter case the slope of the bright end is very poorly constrained, so we assume that the XLF of blue GCs does not differ from the total one, a conclusion which was supported by the analysis presented in P11 for the whole LMXBs population.

In deriving the values presented in Table 3 we assumed a break luminosity of $L_{\mathrm{bk}}=8 \times 10^{37} \mathrm{erg} \mathrm{s}^{-1}$, which is the point where the XLF of individually detected sources starts to deviate from a simple power law (see the solid and dot-dashed lines in Fig. 3). It is possible that $L_{\mathrm{bk}}$ is actually fainter than this value, and that the flattening of the faint end of the XLF in Fig. 3 is due to the completeness limit of our observations (since we are missing an increasing fraction of sources at low fluxes). In this case, however, our conclusion about the flattening of the faint end slope of the intinsic XLF (as opposed to the observed one based on individual detections) at very low luminosities, would be strengthened, since this would imply that a larger fraction of the X-ray flux detected in our stacking experiment is produced in relatively bright LMXBs just below the individual detection threshold. As a result the faint end of the XLF would need to be even shallower, if not with a (differential) positive slope, to compensate for the additional flux near the completeness limit.

We note that a deviation from a simple power law also seems to be present at the bright end $\left(L_{\mathrm{X}} \gtrsim 2 \times 10^{38} \mathrm{erg} \mathrm{s}^{-1}\right)$, where the XLF appears slightly steeper than the model. Given the low statistics it is difficult to tell whether this feature is real, but the same feature is present in the total sample as well (as discussed below in more detail); if confirmed, this would agree with the finding by Kim \& Fabbiano (2010) that old ellipticals tend to have a lower fraction of bright LMXBs, compared to younger galaxies, and thus steeper XLFs above a few $\times 10^{38} \mathrm{erg} \mathrm{s}^{-1}$.

More care is needed to interpret the results derived for the total sample since, although in principle we could expect more robust constrains due to the larger statistics, the $60^{\prime \prime}-240^{\prime \prime}$ region is severely affected by incompleteness issues. We first note that the XLF fails to follow a single power-law down to $L_{X} \sim 8 \times$ $10^{37} \mathrm{erg} \mathrm{s}^{-1}$ as in the case of the restricted sample: the same ML fitting approach used above in this case suggests that the XLF already bends at $L_{\mathrm{X}} \sim 3 \times 10^{38} \mathrm{erg} \mathrm{s}^{-1}$. Since at this luminosity the $\mathrm{XLF}$ is unlikely to be incomplete, as shown in P11 (see their Fig. 6 where the corrected and uncorrected XLF are shown), the data seem to confirm the presence of a high-luminosity break as observed in the restricted sample. We thus require a more complex model to fit the XLF of individually detected sources; we adopt a double power law with a high luminosity break (as opposed to the low-luminosity one discussed earlier) as done in other works (e.g., Sarazin et al. 2001; Kim et al. 2009) and obtain a (very high) slope of $\beta_{\mathrm{VH}}=-3 \pm 0.5$ above the high-luminosity break and $\beta_{\mathrm{H}}=-1.6 \pm 0.4$ below it down to 
$L_{\mathrm{bk}}=8 \times 10^{37} \mathrm{erg} \mathrm{s}^{-1}$. We also note that the position of a lowluminosity break is now more poorly constrained since incompleteness effects become increasingly more important at faint luminosities and part of the XLF flattening is due to incompleteness. Fixing the low-luminosity break to $L_{\mathrm{bk}}=8 \times 10^{37} \mathrm{erg} \mathrm{s}^{-1}$, the stacked flux reported in Table 2 (plus the contribution of the detected sources below the break) implies that the slope of the faint end is $\beta_{L}=-1.46$ with the $3 \sigma$ limits presented in Table 3. This confirms the flattening of the XLF at faint luminosities already found using the restricted sample. Since the slope of the $\mathrm{XLF}$ in the range $8 \times 10^{37} \mathrm{erg} \mathrm{s}^{-1}<L_{\mathrm{X}}<3 \times 10^{38} \mathrm{erg} \mathrm{s}^{-1}$ represents a lower limit to the actual XLF slope, this result is a conservative estimate; the statistical errors quoted here do not account for the systematic uncertainties due to the unknown luminosity of the break nor for the fact that some of the stacked flux may come from sources with luminosity above the $8 \times 10^{37} \mathrm{erg} \mathrm{s}^{-1}$ limit. These considerations make the results for the restricted sample more stringent than the ones for the total set.

\section{Discussion and conclusions}

The results presented in the previous sections show that NGC 1399 possesses a population of LMXBs with luminosities below the detection threshold of individual sources. To be more precise, we are able to verify the presence of such populations inside GCs (which host the majority of LMXBs in this galaxy, see $\mathrm{P} 11$ ), but it is very likely that follow up observations, reaching fainter detection limits will also be able to detect these populations in the field. This result is in agreement with the direct detection of faint LMXBs by Kim et al. (2006b, 2009); Fabbiano et al. (2007); and Zhang et al. (2011) in several other nearer ellipticals (Maffei 1, Centaurus A, NGC 3379, NGC 4697, and NGC 4278) as well as by Voss \& Gilfanov (2007); Revnistev et al. (2008); and Zhang et al. (2011) for the Milky Way, M 31, and M 81. On the other hand, Vulic et al. (2012), who used a stacking method similar to our own, found no evidence of a faint XRB population in M 51 stellar clusters, even considering old clusters similar to GCs; however, it is not clear how these stellar clusters directly compare with the compact GCs found in ellipticals which are more likely to produce close binaries as a result of dynamical effects and high central densities (for instance Vulic and collaborators do not detect a dependence on GC luminosity as the one found in Sivakoff et al. 2008 or P11). Furthermore, it is difficult to estimate the luminosity range covered in their stacking analysis, since the completeness limit of the detected sources that they exclude from the stacking process is not explicitly reported; thus, if their stacking includes only sources at very low luminosities $\left(<10^{35} \mathrm{erg} \mathrm{s}^{-1}\right.$ as stated in the abstract $)$ their data could be consistent with ours if our stacked signal comes from XRBs in the range $10^{35}-10^{37} \mathrm{erg} \mathrm{s}^{-1}$. The optical catalog used by Vulic and collaborators is also deeper than ours (the NGC 1399 color-selected GC sample probes $M_{V}<-7.5$, see P11) which may result in a dilution of the stacked sample if LMXBs are hosted primarily by bright clusters (as generally observed for GCs).

Our analysis shows that the faint GC-LMXBs population has similar properties to its bright counterpart. In particular, we observe that red GCs have an average X-ray luminosity $\sim 3$ times larger than blue GCs $(2.5 \pm 1.0$ for the total sample and $4.1 \pm 2.5$ for the restricted one). Assuming a similar intrinsic average luminosity distribution for all LMXBs, we can interpret this result concluding that red GCs are 3 times more likely to host faint LMXBs than blue ones, as already observed at brighter $\mathrm{X}$-ray luminosities in many recent works (see references in
Sect. 1); alternatively, assuming the same likelihood ratio, faint LMXBs have similar average luminosities in red and blue GCs. These findings thus suggest that the same dynamical and chemical effects, which affect the formation and evolution of bright LMXBs, influence the properties of the entire GC-LMXBs population. It is intriguing that a similar abundance ratio/average luminosity holds at every luminosity since at high and low $L_{X}$ the formation of close binaries follows different paths and the donor star is likely to be different (Fragos et al. 2009; Ivanova 2013). For $L_{X}<2 \times 10^{37}$ magnetic braking in main-sequence stars is the most likely cause of the overabundance of LMXBs in red GCs (Ivanova et al. 2006), while at brighter luminosities the metallicity influences the evolution of red-giant donors (Ivanova et al. 2012). Since our stacked samples can, in principle, include sources up to $L_{\mathrm{X}}<8 \times 10^{37} \mathrm{erg} \mathrm{s}^{-1}$ (our individual source completeness limit in the restricted sample), we are probing both regimes above and below the transition luminosity, which may explain why we retrieve a similar ratio as for brighter LMXBs, if our stacked luminosity is dominated by sources just below the individual detection threshold. However we point out that our result is consistent with Kim et al. (2013) who find that even for $10^{36}<L_{\mathrm{X}}<2 \times 10^{37} \mathrm{erg} \mathrm{s}^{-1}$ the red vs blue ratio is in the range $1.2-3.7$ in a stacked sample of elliptical galaxies in Virgo and Fornax clusters. One more alternative is that LMXBs in red GCs are simply 3 times more luminous on average than their blue GC counterparts; we note, however, that this effect is not observed at brighter luminosities where LMXBs are individually detected and their luminosities and spectrum appear consistent within the two subpopulations (see Kim et al. 2006a; Paolillo et al. 2011). For the reasons discussed above, this scenario appears less likely as it would imply a difference in the type of faint LMXBs hosted by red and blue GCs, which should also mimic the difference observed in their brighter counterparts.

Our stacking experiment indicates that the XLF of LMXBs flattens at low luminosities in NGC 1399, as already found in other nearer galaxies (Voss \& Gilfanov 2006; Fabbiano et al. 2007; Kim et al. 2009; Voss et al. 2009; Zhang et al. 2011) through both direct X-ray binary detection and stacking analysis. On the nature of this break there are various interpretations: for instance, the change of the binary braking mechanism from magnetic stellar winds at high luminosity to gravitational radiation at low luminosity (Postnov \& Kuranov 2005), the transition from LMXBs with predominantly giant donors to binaries with predominantly main-sequence donors (Revnistev et al. 2011), or the presence at low luminosities of a bump, related to contribution of the red giant LMXBs population (Kim et al. 2009). If, as suggested by these works, the XLF of field LMXBs has instead a different shape with steeper slope, this result would argue in favor of a separate formation and/or evolutionary path for GC and field sources (see, e.g, the discussion in Kim et al. 2009).

The hypothesis that LMXBs in GCs and in the field are formed independently (at least most of them) and may represent separate populations is also supported by several studies of spatial profile and specific frequency $S_{N}$ (e.g., Irwin 2006; Kundu et al. 2007; Paolillo et al. 2011). In this respect we point out that in Paolillo et al. (2011) we discussed the link between LMXB abundance in GCs and GC specific frequency (see their Fig. 18); in that case we had to extrapolate the NGC 1399 LF down to $L_{X}=10^{37} \mathrm{erg} \mathrm{s}^{-1}$ to compare with the results of Kim et al. (2009) for NGC 3379, NGC 4697, and NGC 4278. The results shown here indicate that in such a comparison we have to use the lower limits for NGC 1399, corresponding to a broken power-law XLF, thus reducing the disagreement between NGC 1399 and other galaxies of similar $S_{N}$. This indicates that 
the large number of LMXBs in NGC 1399 is consistent with simple statistical fluctuations in the average elliptical galaxy population, a result confirmed by the comparison made by Mineo et al. (2014) at brighter luminosities (see their Fig. 10). On the other hand the same argument would further reduce the dependence of the abundance of field LMXBs on $S_{N}$, thus arguing against a GC origin of field LMXBs.

An alternative explanation of the shape of the GC XLF attributes the flattening at low luminosity just to a bias induced by the presence of multiple LMXBs in the most massive GCs; this effect would remove sources from the faint-end of the XLF and increase the number of bright counterparts. While it is plausible that some of the X-ray bright GCs contain multiple binaries (we find evidence of at least one GC in NGC 1399 in P11), variability measurements suggest that a significant fraction of GCs are dominated by a single XRB both in NGC 1399 (P11) and in other ellipticals (see, e.g, the discussion in Kim et al. 2009).

Finally, we found evidence that the XLF may not be well represented by a single power-law model over the entire luminosity range above the low-luminosity break, and that a second break is required at $L_{\mathrm{X}} \sim 3 \times 10^{38} \mathrm{erg} \mathrm{s}^{-1}$. This result agrees with the model proposed in earlier works by, e.g., Sarazin et al. (2001) and Kim et al. (2009) and may confirm that old ellipticals tend to have a lower fraction of bright LMXBs compared to younger galaxies (Kim \& Fabbiano 2010). This effect was not observed in P11 most likely because of the contamination of background sources (AGNs) which becomes more relevant in the galaxy outskirts. In the present study the contamination is reduced by the smaller surveyed area and the use of color-selected GC subsamples: we estimate, based on extrapolation from deep X-ray counts (see discussion in P11), $<1$ contaminant source over the small annuli considered here. Such contaminants would again strengthen our result about the flattening of the XLF at faint luminosities, as do all other possible systematics discussed in the previous sections.

Deeper Chandra observations will allow us in the future to directly probe the faint LMXBs population, and confirm the results discussed above.

Acknowledgements. M.P. acknowledges support from the FARO 2011 project from the University Federico II of Naples. He also thanks the International Academic Exchange Fund of the "Vicerrectoría Académica" at the Pontificia Universidad Catolica in Santiago, and the Department of Astronomy and Astrophysics for providing travel and lodging support. G.F. is grateful for the hospitality of the Aspen Center for Physics supported by NSF grant No.1066293i.

\section{References}

Angelini, L., Loewenstein, M., \& Mushotzky, R. F. 2001, ApJ, 557, L35 Bassino, L. P., Faifer, F. R., Forte, J. C., et al. 2006, A\&A, 451, 789
Bellazzini, M., Pasquali, A., Federici, L., Ferraro, F. R., \& Pecci, F. F. 1995, ApJ, 439, 687

Bertin, E. \& Arnouts, S. 1996, A\&AS, 117, 393

Boroson, B., Kim, D.-W., \& Fabbiano, G. 2011, ApJ, 729, 12

Brescia, M., Cavuoti, S., Paolillo, M., Longo, G., \& Puzia, T. 2012, MNRAS, 2318

Broos, P. S., Townsley, L. K., Feigelson, E. D., et al. 2010, APJ, 714, 1582

Clauset, A., Shalizi, C. R., \& Newman, M. E. J. 2009, SIAM Rev., 51, 661

de Vaucouleurs, G., de Vaucouleurs, A., Corwin, H. G., et al. 1991, Third Reference Catalogue of Bright Galaxies, Vols. I-III (Springer-Verlag)

Dunn, L. P., \& Jerjen, H. 2006, AJ, 132, 1384

Fabbiano, G. 1989, ARA\&A, 27, 87

Fabbiano, G. 2006, ARA\&A, 44, 323

Fabbiano, G., Brassington, N. J., Zezas, A., et al. 2007, unpublished [arXiv: 0710.5126]

Fragos, T., Lehmer, B., Tremmel, M., et al. 2013, ApJ, 764, 41

Gilfanov, M. 2004, MNRAS, 349, 146

Grimm, H.-J., Gilfanov, M., \& Sunyaev, R. 2002, A\&A, 391, 923

Fragos, T., Kalogera, V., Willems, B., et al. 2009, ApJ, 702, L143

Freeman, P. E., Kashyap, V., Rosner, R., \& Lamb, D. Q. 2002, ApJS, 138, 185

Hornschemeier, A. E., Heckman, T. M., Ptak, A. F., et al. 2005, AJ, 129, 86

Humphrey, P. J., \& Buote, D. A. 2008, ApJ, 689, 983

Irwin, J. 2006, in Proc. The X-ray Universe 2005, ESA SP, 604, 455

Ivanova, N. 2006, ApJ, 636, 979

Ivanova, N. 2013, Mèm. Soc. Astron. It., 84, 123

Ivanova, N., Fragos, T., Kim, D.-W., et al. 2012, ApJ, 760, L24

Jordán, A., Côté, P., Ferrarese, L., et al. 2004, ApJ, 613, 279

Kim, D.-W., \& Fabbiano, G. 2004, ApJ, 611, 846

Kim, D.-W., \& Fabbiano, G. 2010, ApJ, 721, 1523

Kim, D.-W., Fabbiano, G., Kalogera, V., et al. 2006b, ApJ, 652, 1090

Kim, D.-W., Fabbiano, G., Brassington, N. J., et al. 2009, ApJ, 703, 829

Kim, D.-W., Fabbiano, G., Ivanova, N., et al. 2013, ApJ, 764, 98

Kim, E., Kim, D.-W., Fabbiano, G., et al. 2006a, ApJ, 647, 276

Koekemoer, A. M., Fruchter, A. S., Hook, R., \& Hack, W. 2002, HST Calibration Workshop, 337

Kundu, A., Maccarone, T. J., \& Zepf, S. E. 2002, ApJ, 574, L5

Kundu, A., Zepf, S. E., Hempel, M., et al. 2005, ApJ, 634, L41

Kundu, A., Maccarone, T. J., \& Zepf, S. E. 2007, ApJ, 662, 525

Lehmer, B. D., Alexander, D. M., Bauer, F. E., et al. 2010, ApJ, 724, 559

Mineo, S., Gilfanov, M., \& Sunyaev, R. 2012, MNRAS, 419, 2095

Mineo, S., Fabbiano, G., D’Abrusco, R., et al. 2014, ApJ, 780, 132

Newman, M. E. J. 2005, Contemp. Phys. 46, 323

Paolillo, M., Puzia, T. H., Goudfrooij, P., et al. 2011, ApJ, 736, 90 (P11)

Postnov, K. A., \& Kuranov, A. G. 2005, Astron. Lett., 31, 7

Puzia, T. H., Paolillo, M., Goudfrooij, P., et al. 2014, ApJ, 786, 78

Ranalli, P., Comastri, A., \& Setti, G. 2003, A\&A, 399, 39

Revnivtsev, M., Lutovinov, A., Churazov, E., et al. 2008, A\&A, 491, 209

Revnistev, M., Postnov, K., Kuranov, A. \& Ritter, H. 2011, A\&A, 526, A94

Sarazin, C. L., Irwin, J. A., \& Bregman, J. N. 2001, ApJ, 556, 533

Sarazin, C. L., Kundu, A., Irwin, J. A., et al. 2003, ApJ, 595, 743

Schuberth, Y., Richtler, T., Hilker, M., et al. 2010, A\&A, 513, A52

Sivakoff, G. R., Jordàn, A., Sarazin, C. L., et al. 2007, ApJ, 660, 1246

Vanbeveren, D., Mennekens, N., \& De Greve, J. P. 2012, ApJ, 707, 1361

Voss, R., \& Gilfanov, M. 2006, A\&A, 447, 71

Voss, R., \& Gilfanov, M. 2007, A\&A, 468, 49

Voss, R., Gilfanov, M., Sivakoff, G. R., et al. 2009, ApJ, 701, 474

Vulic, N., Barmby, P., \& Gallagher, S. C. 2013, ApJ, 763, 96

Zhang, Z., Gilfanov, M., Voss, R., et al. 2011, A\&A, 533, A33

Zhang, Z., Gilfanov, M., \& Bogdán, Á. 2012, A\&A, 546, A36 